Lidia Typańska-Czajka

\title{
TWO INFINITE SEQUENCES OF PRE-MAXIMAL EXTENSIONS OF THE RELEVANT LOGIC E
}

\begin{abstract}
The only maximal extension of the logic of relevant entailment $\mathbf{E}$ is the classical logic CL. A logic $L \subseteq[\mathbf{E}, \mathbf{C L}]$ called pre-maximal if and only if $L$ is a coatom in the interval $[\mathbf{E}, \mathbf{C L}]$. We present two denumerable infinite sequences of premaximal extensions of the logic $\mathbf{E}$. Note that for the relevant logic $\mathbf{R}$ there exist exactly three pre-maximal logics, i.e. coatoms in the interval $[\mathbf{R}, \mathbf{C L}]$.
\end{abstract}

Keywords: relevant logic, non-classical logics, lattice, universal algebra.

\section{Preliminaries}

Let $F O R$ be the set of all the propositional formulae built up from the propositional variables $p, q, r, p_{1} \ldots$ using the connectives $\neg, \wedge, \vee$ and $\rightarrow$. The first information about the logic of relevant entailment $\mathbf{E}$ can be found in [8]. The logic $\mathbf{E}$ is defined as a subset of the set FOR. $\mathbf{E}$ consists of formulae provable using the following list of axiom schemes:

$E 1 \phi \rightarrow \phi$,

$E 2(\phi \rightarrow \psi) \rightarrow((\psi \rightarrow \chi) \rightarrow \phi \rightarrow \chi))$,

$E 3((\phi \rightarrow \phi) \rightarrow \psi) \rightarrow \psi$,

$E 4(\phi \rightarrow(\phi \rightarrow \psi)) \rightarrow(\phi \rightarrow \psi)$,

E5 $\phi \wedge \psi \rightarrow \phi$,

$E 6 \phi \wedge \psi \rightarrow \psi$,

$E 7(\phi \rightarrow \psi) \wedge(\phi \rightarrow \chi) \rightarrow(\phi \rightarrow \psi \wedge \chi)$, 


$$
\begin{aligned}
E 8 & \phi \rightarrow \phi \vee \psi, \\
E 9 & \psi \rightarrow \phi \vee \psi, \\
E 10 & (\phi \rightarrow \psi) \wedge(\chi \rightarrow \psi) \rightarrow(\phi \vee \chi \rightarrow \psi), \\
E 11 & (\phi \wedge(\psi \vee \chi)) \rightarrow((\phi \wedge \psi) \vee \chi), \\
E 12 & (\phi \rightarrow \neg \psi) \rightarrow(\psi \rightarrow \neg \phi), \\
E 13 & \neg \neg \phi \rightarrow \phi .
\end{aligned}
$$

by application of the rule of modus ponens $(M P: \phi \rightarrow \psi, \phi / \psi)$ and the rule of adjunction $(A D: \phi, \psi / \phi \wedge \psi)$.

The definitions of proof and the metalogical are standard one.

There exists an equivalent version of the logic $\mathbf{E}$ with the same set of axioms, based on the substitution rule.

If we extend the logic $\mathbf{E}$ by adding the axiom

$$
\phi \rightarrow((\phi \rightarrow \psi) \rightarrow \psi),
$$

then we obtain the well known relevant logic $\mathbf{R}$.

The logic $\mathbf{R}$ and the structure of extensions of the logic $\mathbf{R}$ is rather well understood, (see A. R. Anderson, N. D. Belnap [2], W. Dziobiak [6], J. M. Font, G. Rodriguez [5], R. K. Meyer [10], L. L. Maksimowa [7],[8], K. Świrydowicz [11], [12]).

However, the logic $\mathbf{E}$ has not been fully described. One of the basic properties that have been proved is the lack of algebraizability (W.J. Blok and D.L. Pigozzi [4]). Moreover, the logic $\mathbf{E}$ is not structurally complete (see J.M. Dunn, R.M. Meyer [10]). There also exists method of proving theorems of $\mathbf{E}$ introduced by F.Fitch [13].

In addition, it has been shown that there exists exactly three premaximal extension of the logic $\mathbf{R}$, i.e. extensions for which the only extension is the classical logic (see K. Świrydowicz). In the following manuscript we show that there exists infinitely many pre-maximal extensions of the $\operatorname{logic} \mathbf{E}$. 
Two Infinite Sequences of Pre-Maximal Extensions of the Relevant Logic E 31

\subsection{Syntactical matters}

LEMMA 1. The formulae listed below are theses of $\boldsymbol{E}$ :

$(t 1)(p \rightarrow q) \rightarrow((r \rightarrow s) \rightarrow((s \rightarrow p) \rightarrow(r \rightarrow q)))$,

$(t 2)(p \rightarrow q) \wedge(r \rightarrow s) \rightarrow((p \wedge r) \rightarrow(q \wedge s))$,

$(t 3)(p \rightarrow q) \wedge(r \rightarrow s) \rightarrow((p \vee r) \rightarrow(q \vee s))$,

$(t 4)(p \rightarrow q) \rightarrow(\neg q \rightarrow \neg p)$,

$(t 5)(p \wedge(p \rightarrow q)) \rightarrow q$,

(t6) $(p \rightarrow \neg \neg p)$,

$(t 7)((p \wedge q) \vee(p \wedge r)) \leftrightarrow(p \wedge(q \vee r))$, where $\leftrightarrow$ denotes two implications

Proof: Use the Fitch-style proofs.

Lemma 2. Let $\phi\left(p_{1}, \ldots, p_{n}\right)$ be a formula constructed using variables $p_{1}, \ldots, p_{n}$. Then

$\vdash_{E} \phi\left(p_{1}, \ldots, p_{n}\right) \Longleftrightarrow \vdash_{E}\left(p_{1} \rightarrow p_{1}\right) \wedge \ldots \wedge\left(p_{n} \rightarrow p_{n}\right) \rightarrow \phi\left(p_{1}, \ldots, p_{n}\right)$

Next we can prove the following lemma

Lemma 3. $\quad \vdash_{E} \phi \Longleftrightarrow \vdash_{E}\left(\phi_{1} \rightarrow \phi_{1}\right) \wedge \ldots \wedge\left(\phi_{n} \rightarrow \phi_{n}\right) \rightarrow \phi$ for some subformulae $\phi_{1}, \ldots, \phi_{n}$ of the formula $\phi$. In particular,

$$
\vdash_{E}(\phi \rightarrow \psi) \Longleftrightarrow \vdash_{E}(\phi \rightarrow \phi) \rightarrow(\phi \rightarrow \psi)
$$

\subsection{Algebraic matters}

Definition 4. An Algebra $\mathbf{A}=\langle A, \wedge, \vee, \rightarrow, \neg\rangle$ is called an E-algebra, if $\langle A, \wedge, \vee\rangle$ is a distributive lattice and the following conditions are satisfied for all $x, y, z \in \mathbf{A}$ :

$(e 1)(x \rightarrow y) \leq((y \rightarrow z) \rightarrow(x \rightarrow z))$,

$(e 2) \quad((x \rightarrow x) \rightarrow y) \leq y$,

$(e 3)(x \rightarrow(x \rightarrow y)) \leq(x \rightarrow y)$,

$(e 4)(x \rightarrow y) \wedge(v \rightarrow s) \leq((x \wedge v) \rightarrow(y \wedge s))$,

$(e 5)(x \rightarrow y) \wedge(v \rightarrow s) \leq((x \vee v) \rightarrow(y \vee s))$,

(e6) $(x \rightarrow \neg y) \leq(y \rightarrow \neg x)$,

(e7) $x=\neg \neg x$.

In the expressions above, $\leq$ denotes partial order of the lattice $\langle A, \wedge, \vee\rangle)$. The lattice $\langle A, \wedge, \vee\rangle$ of the algebra $\mathbf{A}$ is called lattice of this $\boldsymbol{E}$-algebra. 
Definition 5. A pair $\left\langle\mathbf{A}, \nabla_{\mathbf{A}}\right\rangle$ is called an $\mathbf{E}$-matrix, if $\mathbf{A}$ is an $\mathbf{E}$-algebra, and $\nabla_{A} \subseteq A$ satisfies the condition

$$
x \in \nabla_{A} \Longleftrightarrow\left(x_{1} \rightarrow x_{1}\right) \wedge \ldots \wedge\left(x_{n} \rightarrow x_{n}\right) \leq x
$$

for some $\left(x_{1} \rightarrow x_{1}\right), \ldots,\left(x_{n} \rightarrow x_{n}\right)$. The set $\nabla_{A}$ is called a set of the designated elements of the algebra $\mathbf{A}$.

LEMma 6. The set $\nabla_{A}$ is a filter on $\mathbf{A}$.

Definition 7. Let $\mathbf{A}$ be an E-algebra. The logic $L(\mathbf{A})$ generated by the matrix $\left\langle\mathbf{A}, \nabla_{\mathbf{A}}\right\rangle$ is the set of the formulae which satisfy the following condition:

$$
\phi \in L(\mathbf{A}) \Longleftrightarrow \forall_{h: F O R \rightarrow A} \quad\left(h(\phi) \in \nabla_{\mathbf{A}}\right),
$$

where $h: F O R \rightarrow \mathbf{A}$ is homomorphism.

Definition 8. If $h(\phi) \in \nabla_{\mathbf{A}}$ for any homomorphism $h: F O R \rightarrow \mathbf{A}$, then $\phi$ is called an $\left\langle\mathbf{A}, \nabla_{\mathbf{A}}\right\rangle$-tautology or simply $\mathbf{A}$-tautology.

\section{TheOREM 9. (Completeness of $\mathbf{E})$}

$$
\vdash_{E} \phi \Longleftrightarrow h(\phi) \in \nabla_{\mathbf{A}}
$$

for any $\boldsymbol{E}$-algebra $\mathbf{A}$, and for any homomorphism $h: F O R \rightarrow A$, where $\nabla_{\mathbf{A}}$ is the set of designated elements of $\boldsymbol{A}$.

Proof: $(\Rightarrow)$ Induction on the length of a proof of $\phi$ in $\mathbf{E}$.

$(\Leftarrow)$ Construction of the Lindenbaum algebra of $\mathbf{E}\left(\operatorname{Lind}_{\mathbf{E}}\right)$.

Recall that the Lindenbaum algebra for the logic $\mathbf{E} \operatorname{Lind}_{\mathbf{E}}$ is constructed of the set FOR by the equivalence relation defined by:

$$
\psi \sim \phi \Longleftrightarrow \vdash_{\mathbf{E}} \psi \rightarrow \phi \wedge \vdash_{\mathbf{E}} \phi \rightarrow \psi
$$

The partial order $\leq$ is defined by $\phi / \sim \leq \psi / \sim \Longleftrightarrow \vdash_{\mathbf{E}}(\phi \rightarrow \psi)$.

Lind $_{\mathbf{E}}$ is an E-algebra; in particular:

(*) $\quad \phi / \sim \leq \psi / \sim \Longleftrightarrow(\phi / \sim \rightarrow \psi / \sim) \in \nabla_{\text {Lind }_{\mathbf{E}}}$, ie.

$(* *) \quad x \leq y \Longleftrightarrow(x \rightarrow y) \in \nabla_{\text {Lind }_{\mathbf{E}}}$

We point out that the equivalences $(*)$ and $(* *)$ do not need to hold in each E-algebra. 
Finally, we have

Corollary 10. Let $\vdash_{\mathbf{E}}(\phi \rightarrow \psi)$. Then for each $\mathbf{E}$-algebra $\mathbf{A}$ and for each $h: F O R \rightarrow \mathbf{A}$ the following inequality holds

$$
h(\phi) \leq h(\psi) .
$$

Thus, each E-theorem in the form $\phi \rightarrow \psi$ generates an inequality in each E-algebra.

For a given algebra $\mathbf{A}$ the filter $\nabla_{\mathbf{A}}$ is uniquely defined. Hence, now we show how to differentiate between $\mathbf{E}$-algebras and $\mathbf{E}$-matrices.

LEMma 11. Let $\mathbf{A}$ be an $\boldsymbol{E}$-algebra and $\nabla_{A}=\left\{x \in A: \exists t_{k}\left(t_{k} \leq x\right)\right\}$, where $t_{k}=\bigwedge_{1<i<k}\left(x_{i} \rightarrow x_{i}\right)$ for some elements $a_{i} \in \boldsymbol{A}$ and let $\nabla_{\mathbf{A}} \subseteq \nabla$. Then the relation $\theta(\nabla)$ defined by the equivalence

$$
(x \equiv y) \theta(\nabla) \Longleftrightarrow((x \rightarrow y),(y \rightarrow x) \in \nabla)
$$

is a congruence relation on $\boldsymbol{A}$.

Lemma 12. Let $\theta$ be a congruence relation on the $\boldsymbol{E}$-algebra $\boldsymbol{A}$. Then the set $\nabla(\theta)=\left\{x: \exists y\left(y \in \nabla_{\mathbf{A}}\right) \wedge\left(x \equiv_{\theta} y\right)\right\}$ is a filter and $\nabla_{\mathbf{A}} \subseteq \nabla(\theta)$.

Proof: Easy. (cf. Definition 4)

Let $\mathcal{F}\left(\nabla_{\mathbf{A}}\right)=\left\{\nabla: \nabla\right.$ is a filter and $\left.\nabla_{\mathbf{A}} \subseteq \nabla\right\}$. If $\mathbf{A}$ is an $\mathbf{R}$-algebra, then the lattices $\operatorname{Con}(\mathbf{A})$ and $\mathcal{F}\left(\nabla_{\mathbf{A}}\right)$ are isomorphic. However, if $\mathbf{A}$ is an E-algebra, then $\operatorname{Con}(\mathbf{A})$ and $\mathcal{F}\left(\nabla_{\mathbf{A}}\right)$ do not have to be isomorphic (see W.J. Blok and D. Pigozzi) [4].

Definition 13. An algebra $\mathbf{A}$ is called a simple algebra, if $C o n(\mathbf{A})$ contains exactly two elements.

By Corollary 10 and the definition of E-algebra (refdef:1) we get the following useful lemma.

LEMMA 14. The following inequalities hold in each $\boldsymbol{E}$-algebra:

(1) $x \wedge(x \rightarrow y) \leq y$,

(2) $(\neg x \rightarrow x) \leq x$,

(3) $\left(x \rightarrow\left(\left(y_{1} \rightarrow y_{2}\right) \rightarrow z\right)\right) \leq\left(\left(y_{1} \rightarrow y_{2}\right) \rightarrow(x \rightarrow z)\right)$. 
Lemma 15. Moreover, we have additional useful implications and inequalities:

(i) $x \in \nabla \Longrightarrow x \rightarrow y \leq y$,

(ii) Let $y \rightarrow y=a$. Then $(x \rightarrow y) \leq a \rightarrow(x \rightarrow y)$.

Lemma 16. Let $\boldsymbol{A}$ satisfy the inequality $((x \rightarrow x) \rightarrow(y \rightarrow z)) \leq(y \rightarrow$ $((x \rightarrow x) \rightarrow z))$. Then the following conditions are equivalent

(') $x \leq y \Longleftrightarrow(x \rightarrow y) \in \nabla_{\mathbf{A}}$.

${ }^{(\prime)}(x \rightarrow x) \leq(y \rightarrow z) \Longleftrightarrow y \leq((x \rightarrow x) \rightarrow z)$.

Corollary 17. If A satisfies the inequality $(x \rightarrow x) \rightarrow(y \rightarrow z) \leq(y \rightarrow$ $((x \rightarrow x) \rightarrow z))$, then A satisfies $\left(x_{1} \rightarrow x_{2}\right) \rightarrow\left(\left(y_{1} \rightarrow y_{2}\right) \rightarrow z\right) \leq\left(\left(y_{1} \rightarrow\right.\right.$ $\left.\left.y_{2}\right) \rightarrow\left(\left(x_{1} \rightarrow x_{2}\right) \rightarrow z\right)\right)$.

\section{Two infinite sequences of algebras}

\subsection{Introductory remarks}

In this section we present the construction of two infinite sequences of E-algebras whose lattices are chains. For convenience, we us horizontal notation for chains (i.e., chains are written in a number like-line fashion).

Since all the E-algebras considered below are based on finite chains, hence these algebras have a smallest element (denoted by 0 ) and a greatest element (denoted by 1 ). Moreover, we use $a$, to denote an atom in all E-algebras. In addition, $\nabla=[a)=\{x: a \leq x\}$.

Lemma 18. The following equalities hold in $\boldsymbol{E}$-algebras:

$1 \rightarrow 1=1,0 \rightarrow x=1,1 \rightarrow 0=0,0 \rightarrow 1=1$.

If $\boldsymbol{E}$-algebra $\mathbf{A}$ is a chain, $\nabla_{\mathbf{A}}=[a)$ and $a$ is an atom, then $x \rightarrow 0=$ $0, x \neq 0$, if $x \in \nabla_{\mathbf{A}}$.

Since we examine only $\mathbf{E}$-algebras based on chains and $\nabla_{A}=[a)$, where $a$ is an atom, hence the equality $x \rightarrow 0$ holds for all $x$ in $\mathbf{A}$. LEMma 19. The algebra $\mathbf{2}$ is a subalgebra of each nontrivial $\boldsymbol{E}$-algebra. 


\subsection{Construction of $A_{n}$-algebras}

\section{$A_{0}$-algebras}

Let us consider the following lattice:

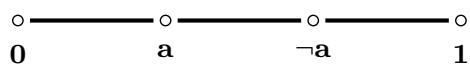

If the lattice of an $\mathbf{E}$-algebra is a 4-elements chain the (operation $\neg$ is obvious here), then the operation $\rightarrow$ must be defined as the following table shows

\begin{tabular}{|c||c|c|c|c|}
\hline$\rightarrow$ & 0 & $a$ & $\neg a$ & 1 \\
\hline \hline 0 & 1 & 1 & 1 & 1 \\
\hline$a$ & 0 & $a$ & & 1 \\
\hline$\neg a$ & 0 & 0 & $a$ & 1 \\
\hline 1 & 0 & 0 & 0 & 1 \\
\hline
\end{tabular}

We observe $a \leq \neg a$, thus $(a \rightarrow \neg a) \in \nabla$, ie. $(a \rightarrow \neg a) \in[a)$, i.e. $a \leq a \rightarrow \neg a$. By the other hand, by the Clavius law $a \rightarrow \neg a \leq \neg a$. Summing it up, $a \leq(a \rightarrow \neg a) \leq \neg a$.

We conclude that the function $\rightarrow$ for $a \rightarrow \neg a$ can be defined in the following three ways:

1. $a \rightarrow \neg a=a$

2. $a \rightarrow \neg a=\neg a$

3. $a \rightarrow \neg a \neq a, a \rightarrow \neg a \neq \neg a$, i.e. $a \rightarrow \neg a$ is $a$ new element different from $a, \neg a$.

If we assume that $a \rightarrow \neg a=a$ or $a \rightarrow \neg a=\neg a$, then we get two distinct $\mathbf{E}$-algebras and the function $\rightarrow$ can be defined as in the following $\rightarrow$-tables:

\begin{tabular}{|c||c|c|c|c|}
\hline$\rightarrow$ & 0 & $a$ & $\neg a$ & 1 \\
\hline \hline 0 & 1 & 1 & 1 & 1 \\
\hline$a$ & 0 & $a$ & $a$ & 1 \\
\hline$\neg a$ & 0 & 0 & $a$ & 1 \\
\hline 1 & 0 & 0 & 0 & 1 \\
\hline
\end{tabular}

\begin{tabular}{|c||c|c|c|c|}
\hline$\rightarrow$ & 0 & $a$ & $\neg a$ & 1 \\
\hline \hline 0 & 1 & 1 & 1 & 1 \\
\hline$a$ & 0 & $a$ & $\neg a$ & 1 \\
\hline$\neg a$ & 0 & 0 & $a$ & 1 \\
\hline 1 & 0 & 0 & 0 & 1 \\
\hline
\end{tabular}

We encourage the reader to prove that the function $\rightarrow$ satisfies the inequalities which define $\mathbf{E}$-algebras. 


\section{$A_{1}$-algebras}

Let $a \rightarrow \neg a \neq a$ and $a \rightarrow \neg a \neq \neg a$; let $(a \rightarrow \neg a):=a_{1}$. Assume that $a_{1} \leq \neg a_{1}$ or $\neg a_{1} \leq a_{1}$, so we have two 6 -elements chains. Thus there exists two possibilities:

1.

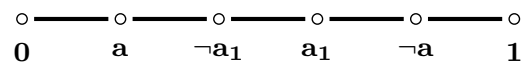

2.

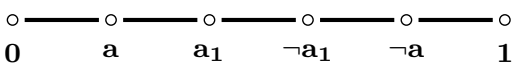

We observe that if we assume that $a_{1} \rightarrow a_{1}=a_{1}$, then the algebrahas its own subalgebra that is different from $\mathbf{2}$. Therefore, we assume that $a_{1} \rightarrow a_{1}=a$. Moreover, we determine the values for some of the elements in $\rightarrow$-table independently of the ordering of $a_{1}$ and $\neg a_{1}$.

1. We observe that $a \leq a \rightarrow a_{1} \leq a_{1}$. By the syllogism, $a \rightarrow \neg a \leq$ $(\neg a \rightarrow \neg a) \rightarrow(a \rightarrow \neg a)$, thus $a_{1} \leq a \rightarrow a_{1}$. Hence $a \rightarrow a_{1}=a_{1}$

2. Similarly, $a \leq a \rightarrow \neg a_{1} \leq \neg a_{1}$. By the syllogism, $a \rightarrow \neg a_{1} \leq$ $\left(\neg a_{1} \rightarrow \neg a\right) \rightarrow(a \rightarrow \neg a)$, thus $a \rightarrow \neg a_{1} \leq a_{1} \rightarrow a_{1}$ so $a \rightarrow \neg a_{1} \leq a$. Therefore $a \rightarrow \neg a_{1}=a$

3. Assume that $\neg a_{1} \leq a_{1}$ (the first chain). It is clear that $a \leq \neg a_{1} \rightarrow$ $a_{1} \leq a_{1}$. By $($ ii $)$ in Lemma $15 \neg a_{1} \rightarrow a_{1} \leq a \rightarrow\left(\neg a_{1} \rightarrow a_{1}\right)$. If we take elements between $a$ and $a_{1}$, then we obtain that $\neg a_{1} \rightarrow a_{1}=a$ or $\neg a_{1} \rightarrow a_{1}=a_{1}$, because other cases lead to a contradiction.

Remark. Assume now that $a_{1} \leq \neg a_{1}$. Thus $a \leq a_{1} \rightarrow \neg a_{1}$. By the syllogism, $a_{1} \rightarrow \neg a_{1} \leq\left(\neg a_{1} \rightarrow \neg a\right) \rightarrow\left(a_{1} \rightarrow \neg a\right)$ and we obtain $a_{1} \rightarrow$ $\neg a_{1} \leq a_{1} \rightarrow a$, i.e. $a_{1} \rightarrow \neg a_{1} \leq 0$, which is a contradiction. We conclude that algebra does not exist.

As a consequence of the reasoning presented above, only the first chain in which $\neg a_{1} \leq a_{1}$ can be the basis of our 6 -element algebras.

We use $\mathbf{A}_{\mathbf{1}}$ to denote the algebras based on our 6-element chain. For simplicity of notation, we omit the first and the last lines and the first and the last column in this $\rightarrow$-tables (cf. Lemma 18).

We infer that $\rightarrow$-tables for $\mathbf{A}_{\mathbf{1}}$-algebras: 
Two Infinite Sequences of Pre-Maximal Extensions of the Relevant Logic $\mathbf{E}$

\begin{tabular}{|c||c|c|c|c|}
\hline$\rightarrow$ & $a$ & $\neg a_{1}$ & $a_{1}$ & $\neg a$ \\
\hline \hline$a$ & $a$ & $a$ & $a_{1}$ & $a_{1}$ \\
\hline$\neg a_{1}$ & 0 & $a$ & $a$ & $a_{1}$ \\
\hline$a_{1}$ & 0 & 0 & $a$ & $a$ \\
\hline$\neg a$ & 0 & 0 & 0 & $a$ \\
\hline
\end{tabular}

\begin{tabular}{|c||c|c|c|c|}
\hline$\rightarrow$ & $a$ & $\neg a_{1}$ & $a_{1}$ & $\neg a$ \\
\hline \hline$a$ & $a$ & $a$ & $a_{1}$ & $a_{1}$ \\
\hline$\neg a_{1}$ & 0 & $a$ & $a_{1}$ & $a_{1}$ \\
\hline$a_{1}$ & 0 & 0 & $a$ & $a$ \\
\hline$\neg a$ & 0 & 0 & 0 & $a$ \\
\hline
\end{tabular}

We use $\mathbf{A}_{\mathbf{1}, \mathbf{a}}$ to denote the algebra in which $\neg a_{1} \rightarrow a_{1}=a$; in $\mathbf{A}_{\mathbf{1}, \mathbf{a}_{\mathbf{1}}}$, $\neg a_{1} \rightarrow a_{1}=a_{1}$.

The Reader can check that these $\mathbf{A}_{\mathbf{1}}$-algebras are $\mathbf{E}$-algebras.

The algebras $\mathbf{A}_{\mathbf{1}, \mathbf{a}}$ and $\mathbf{A}_{\mathbf{1}, \mathbf{a}_{\mathbf{1}}}$ are called $\mathbf{A}_{\mathbf{1}}$-algebras.

\section{$A_{2}$-algebras}

We have $a \leq \neg a_{1} \rightarrow a_{1} \leq a_{1}$. Assume that $\neg a_{1} \rightarrow a_{1} \neq a$ and $\neg a_{1} \rightarrow a_{1} \neq a_{1}$. Let us consider a new element $\neg a_{1} \rightarrow a_{1}:=a_{2}$. Hence we consider an 8-elements chain in which $\neg a_{2} \leq a_{2}$ (the case $a_{2} \leq \neg a_{2}$ is impossible):

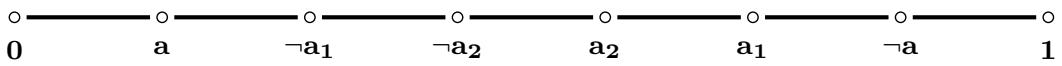

We observe that $a \leq \neg a_{1} \rightarrow a_{2} \leq a_{2}$. By (ii) in Lemma 15, $\neg a_{1} \rightarrow$ $a_{2} \leq a \rightarrow\left(\neg a_{1} \rightarrow a_{2}\right)$. Therefore, we have two possibilities: $\neg a_{1} \rightarrow a_{2}=a$ or $\neg a_{1} \rightarrow a_{2}=a_{2}$.

As a result, we define two $\mathbf{A}_{\mathbf{2}}$-algebras based on our 8-elements lattice (cf. the picture above). In the first algebra, $\mathbf{A}_{\mathbf{2}, \mathbf{a}}$ we have $\neg a_{1} \rightarrow a_{2}=a$ and in the second algebra $\mathbf{A}_{2, \mathbf{a}_{2}}$ we have $\neg a_{1} \rightarrow a_{2}=a_{2}$.

\begin{tabular}{|c||c|c|c|c|c|c|}
\hline$\rightarrow$ & $a$ & $\neg a_{1}$ & $\neg a_{2}$ & $a_{2}$ & $a_{1}$ & $\neg a$ \\
\hline \hline$a$ & $a$ & $a$ & $a$ & $a_{2}$ & $a_{1}$ & $a_{1}$ \\
\hline$\neg a_{1}$ & 0 & $a$ & $a$ & $a$ & $a_{2}$ & $a_{1}$ \\
\hline$\neg a_{2}$ & 0 & 0 & $a$ & $a$ & $a$ & $a_{2}$ \\
\hline$a_{2}$ & 0 & 0 & 0 & $a$ & $a$ & $a$ \\
\hline$a_{1}$ & 0 & 0 & 0 & 0 & $a$ & $a$ \\
\hline$\neg a$ & 0 & 0 & 0 & 0 & 0 & $a$ \\
\hline
\end{tabular}

\begin{tabular}{|c||c|c|c|c|c|c|}
\hline$\rightarrow$ & $a$ & $\neg a_{1}$ & $\neg a_{2}$ & $a_{2}$ & $a_{1}$ & $\neg a$ \\
\hline \hline$a$ & $a$ & $a$ & $a$ & $a_{2}$ & $a_{1}$ & $a_{1}$ \\
\hline$\neg a_{1}$ & 0 & $a$ & $a$ & $a_{2}$ & $a_{2}$ & $a_{1}$ \\
\hline$\neg a_{2}$ & 0 & 0 & $a$ & $a$ & $a_{2}$ & $a_{2}$ \\
\hline$a_{2}$ & 0 & 0 & 0 & $a$ & $a$ & $a$ \\
\hline$a_{1}$ & 0 & 0 & 0 & 0 & $a$ & $a$ \\
\hline$\neg a$ & 0 & 0 & 0 & 0 & 0 & $a$ \\
\hline
\end{tabular}




\section{$A_{n}$-Algebras}

So far, we only considered chains have with even numbers of elements. In addition, each chain has a smallest element and a greatest element, and it has an element $a$ and an element $\neg a$. The remaining elements are of the form $a_{k}$ and $\neg a_{k}$. Thus all our chains have $2+2+2 k$ elements. If our chain has $2+2+2 k$, then the algebra generated by this chain will be denoted by $\mathbf{A}_{\mathbf{k}}$, for example, algebras $\mathbf{A}_{\mathbf{3}}$ have $2+2+2 \cdot 3$ elements.

Let us generalize the procedure of defining operation $\rightarrow$ for $A_{n}$-chains.

Let us consider the $\mathbf{A}_{\mathbf{n}}$-chain. In fact the algebra $\mathbf{A}_{\mathbf{n}}$ is an 'extension' of the algebra $\mathbf{A}_{\mathbf{n}-\mathbf{1}}$, i.e. the values of the operation $\rightarrow$ on the elements of $\mathbf{A}_{\mathbf{n}}$ and on the elements of $\mathbf{A}_{\mathbf{n}-\mathbf{1}}$ are exactly the same with the exception of the element $\neg a_{1} \rightarrow a_{n-1}$ and its negation; $\neg a_{1} \rightarrow a_{n-1}$ in $\mathbf{A}_{\mathbf{n}}$ equals $a$ or $a_{n-1}$, but equals $a_{n}$ in $\mathbf{A}_{\mathbf{n}}$. We obtain

1. $\neg a_{1} \rightarrow a_{n-1}=a$ in $\mathbf{A}_{\mathbf{n}-\mathbf{1}, \mathbf{a}}$

2. $\neg a_{1} \rightarrow a_{n-1}=a_{n-1}$ in $\mathbf{A}_{\mathbf{n}-\mathbf{1}, \mathbf{a}_{\mathbf{n}-\mathbf{1}}}$

3. $\neg a_{1} \rightarrow a_{n-1}=a_{n}$ in $\mathbf{A}_{\mathbf{n}}$.

In other words, in the algebra $\mathbf{A}_{\mathbf{n}}$ the element $\neg a_{1} \rightarrow a_{n-1}=a_{n}$ differs from $a$ and $a_{n-1}$. Thus we must consider $A_{n}$-chain where $\neg a_{n} \leq a_{n}$ (the case $a_{n} \leq \neg a_{n}$ is impossible):

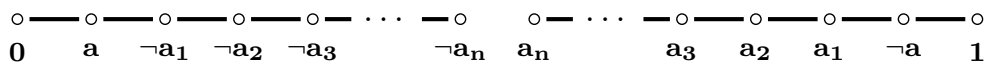

In fact there are two $\mathbf{A}_{\mathbf{n}}$-algebras, i.e. the first, $\mathbf{A}_{\mathbf{n}, \mathbf{a}}$, in which $\neg a_{1} \rightarrow$ $a_{n}=a$ : 
Two Infinite Sequences of Pre-Maximal Extensions of the Relevant Logic $\mathbf{E}$

\begin{tabular}{|c||c|c|c|c|c|c|c|c|c|c|c|c|}
\hline$\rightarrow$ & $a$ & $\neg a_{1}$ & $\neg a_{2}$ & $\ldots$ & $\neg a_{n-1}$ & $\neg a_{n}$ & $a_{n}$ & $a_{n-1}$ & $\ldots$ & $a_{2}$ & $a_{1}$ & $\neg a$ \\
\hline \hline$a$ & $a$ & $a$ & $a$ & $\ldots$ & $a$ & $a$ & $a_{n}$ & $a_{n-1}$ & $\ldots$ & $a_{2}$ & $a_{1}$ & $a_{1}$ \\
\hline$\neg a_{1}$ & 0 & $a$ & $a$ & $\ldots$ & $a$ & $a$ & $a$ & $a_{n}$ & $\ldots$ & $a_{3}$ & $a_{2}$ & $a_{1}$ \\
\hline$\neg a_{2}$ & 0 & 0 & $a$ & $\ldots$ & $a$ & $a$ & $a$ & $a$ & $\ldots$ & $a$ & $a_{3}$ & $a_{2}$ \\
\hline$\vdots$ & $\vdots$ & $\vdots$ & $\vdots$ & $\ddots$ & $\vdots$ & $\vdots$ & $\vdots$ & $\vdots$ & $\ddots$ & $\vdots$ & $\vdots$ & $\vdots$ \\
\hline$\neg a_{n-1}$ & 0 & 0 & 0 & $\ldots$ & $a$ & $a$ & $a$ & $a$ & $\ldots$ & $a$ & $a_{n}$ & $a_{n-1}$ \\
\hline$\neg a_{n}$ & 0 & 0 & 0 & $\ldots$ & 0 & $a$ & $a$ & $a$ & $\ldots$ & $a$ & $a$ & $a_{n}$ \\
\hline$a_{n}$ & 0 & 0 & 0 & $\ldots$ & 0 & 0 & $a$ & $a$ & $\ldots$ & $a$ & $a$ & $a$ \\
\hline$a_{n-1}$ & 0 & 0 & 0 & $\ldots$ & 0 & 0 & 0 & $a$ & $\ldots$ & $a$ & $a$ & $a$ \\
\hline$\vdots$ & $\vdots$ & $\vdots$ & $\vdots$ & $\ddots$ & $\vdots$ & $\vdots$ & $\vdots$ & $\vdots$ & $\ddots$ & $\vdots$ & $\vdots$ & $\vdots$ \\
\hline$a_{2}$ & 0 & 0 & 0 & $\ldots$ & 0 & 0 & 0 & 0 & $\ldots$ & $a$ & $a$ & $a$ \\
\hline$a_{1}$ & 0 & 0 & 0 & $\ldots$ & 0 & 0 & 0 & 0 & $\ldots$ & 0 & $a$ & $a$ \\
\hline$\neg a$ & 0 & 0 & 0 & $\ldots$ & 0 & 0 & 0 & 0 & $\ldots$ & 0 & 0 & $a$ \\
\hline
\end{tabular}

and the second, $\mathbf{A}_{\mathbf{n}, \mathbf{a}_{\mathbf{n}}}$, in which $\neg a_{1} \rightarrow a_{n}=a_{n}$ :

\begin{tabular}{|c||c|c|c|c|c|c|c|c|c|c|c|c|}
\hline$\rightarrow$ & $a$ & $\neg a_{1}$ & $\neg a_{2}$ & $\ldots$ & $\neg a_{n-1}$ & $\neg a_{n}$ & $a_{n}$ & $a_{n-1}$ & $\ldots$ & $a_{2}$ & $a_{1}$ & $\neg a$ \\
\hline \hline$a$ & $a$ & $a$ & $a$ & $\ldots$ & $a$ & $a$ & $a_{n}$ & $a_{n-1}$ & $\ldots$ & $a_{2}$ & $a_{1}$ & $a_{1}$ \\
\hline$\neg a_{1}$ & 0 & $a$ & $a$ & $\ldots$ & $a$ & $a$ & $a_{n}$ & $a_{n}$ & $\ldots$ & $a_{3}$ & $a_{2}$ & $a_{1}$ \\
\hline$\neg a_{2}$ & 0 & 0 & $a$ & $\ldots$ & $a$ & $a$ & $a$ & $a$ & $\ldots$ & $a$ & $a_{3}$ & $a_{2}$ \\
\hline$\vdots$ & $\vdots$ & $\vdots$ & $\vdots$ & $\ddots$ & $\vdots$ & $\vdots$ & $\vdots$ & $\vdots$ & $\ddots$ & $\vdots$ & $\vdots$ & $\vdots$ \\
\hline$\neg a_{n-1}$ & 0 & 0 & 0 & $\ldots$ & $a$ & $a$ & $a$ & $a$ & $\ldots$ & $a$ & $a_{n}$ & $a_{n-1}$ \\
\hline$\neg a_{n}$ & 0 & 0 & 0 & $\ldots$ & 0 & $a$ & $a$ & $a$ & $\ldots$ & $a$ & $a_{n}$ & $a_{n}$ \\
\hline$a_{n}$ & 0 & 0 & 0 & $\ldots$ & 0 & 0 & $a$ & $a$ & $\ldots$ & $a$ & $a$ & $a$ \\
\hline$a_{n-1}$ & 0 & 0 & 0 & $\ldots$ & 0 & 0 & 0 & $a$ & $\ldots$ & $a$ & $a$ & $a$ \\
\hline$\vdots$ & $\vdots$ & $\vdots$ & $\vdots$ & $\ddots$ & $\vdots$ & $\vdots$ & $\vdots$ & $\vdots$ & $\ddots$ & $\vdots$ & $\vdots$ & $\vdots$ \\
\hline$a_{2}$ & 0 & 0 & 0 & $\ldots$ & 0 & 0 & 0 & 0 & $\ldots$ & $a$ & $a$ & $a$ \\
\hline$a_{1}$ & 0 & 0 & 0 & $\ldots$ & 0 & 0 & 0 & 0 & $\ldots$ & 0 & $a$ & $a$ \\
\hline$\neg a$ & 0 & 0 & 0 & $\ldots$ & 0 & 0 & 0 & 0 & $\ldots$ & 0 & 0 & $a$ \\
\hline
\end{tabular}

\section{$A_{n+1}$-algebras}

The construction of the algebras $\mathbf{A}_{\mathbf{n}+\mathbf{1}}$ is very similar. As in the case of $\mathbf{A}_{\mathbf{n}}$-algebras, we observe that $\rightarrow$ can satisfy the conditions: 
1. $\neg a_{1} \rightarrow a_{n+1}=a$

2. $\neg a_{1} \rightarrow a_{n+1}=a_{n+1}$

3. $\neg a_{1} \rightarrow a_{n+1} \neq a$ and $\neg a_{1} \rightarrow a_{n+1} \neq a_{n+1}$

Hence, we obtain the following $A_{n+1}$-chain (the case $a_{n+1} \leq \neg a_{n+1}$ is impossible):

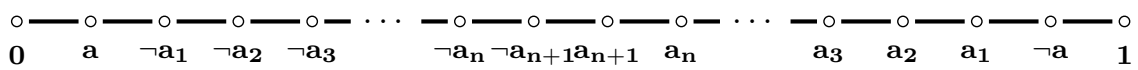

Thus we have two $\mathbf{A}_{\mathbf{n}+\mathbf{1}}$-algebras, i.e. the first, $\mathbf{A}_{\mathbf{n}+\mathbf{1}, \mathbf{a}}$, in which $\neg a_{1} \rightarrow$ $a_{n+1}=a$ :

\begin{tabular}{|c||c|c|c|c|c|c|c|c|c|c|c|c|}
\hline$\rightarrow$ & $a$ & $\neg a_{1}$ & $\neg a_{2}$ & $\ldots$ & $\neg a_{n}$ & $\neg a_{n+1}$ & $a_{n+1}$ & $a_{n}$ & $\ldots$ & $a_{2}$ & $a_{1}$ & $\neg a$ \\
\hline \hline$a$ & $a$ & $a$ & $a$ & $\ldots$ & $a$ & $a$ & $a_{n+1}$ & $a_{n-1}$ & $\ldots$ & $a_{2}$ & $a_{1}$ & $a_{1}$ \\
\hline$\neg a_{1}$ & 0 & $a$ & $a$ & $\ldots$ & $a$ & $a$ & $a$ & $a_{n+1}$ & $\ldots$ & $a_{3}$ & $a_{2}$ & $a_{1}$ \\
\hline$\neg a_{2}$ & 0 & 0 & $a$ & $\ldots$ & $a$ & $a$ & $a$ & $a$ & $\ldots$ & $a$ & $a_{3}$ & $a_{2}$ \\
\hline$\vdots$ & $\vdots$ & $\vdots$ & $\vdots$ & $\ddots$ & $\vdots$ & $\vdots$ & $\vdots$ & $\vdots$ & $\ddots$ & $\vdots$ & $\vdots$ & $\vdots$ \\
\hline$\neg a_{n}$ & 0 & 0 & 0 & $\ldots$ & $a$ & $a$ & $a$ & $a$ & $\ldots$ & $a$ & $a_{n+1}$ & $a_{n-1}$ \\
\hline$\neg a_{n+1}$ & 0 & 0 & 0 & $\ldots$ & 0 & $a$ & $a$ & $a$ & $\ldots$ & $a$ & $a$ & $a_{n+1}$ \\
\hline$a_{n+1}$ & 0 & 0 & 0 & $\ldots$ & 0 & 0 & $a$ & $a$ & $\ldots$ & $a$ & $a$ & $a$ \\
\hline$a_{n}$ & 0 & 0 & 0 & $\ldots$ & 0 & 0 & 0 & $a$ & $\ldots$ & $a$ & $a$ & $a$ \\
\hline$\vdots$ & $\vdots$ & $\vdots$ & $\vdots$ & $\ddots$ & $\vdots$ & $\vdots$ & $\vdots$ & $\vdots$ & $\ddots$ & $\vdots$ & $\vdots$ & $\vdots$ \\
\hline$a_{2}$ & 0 & 0 & 0 & $\ldots$ & 0 & 0 & 0 & 0 & $\ldots$ & $a$ & $a$ & $a$ \\
\hline$a_{1}$ & 0 & 0 & 0 & $\ldots$ & 0 & 0 & 0 & 0 & $\ldots$ & 0 & $a$ & $a$ \\
\hline$\neg a$ & 0 & 0 & 0 & $\ldots$ & 0 & 0 & 0 & 0 & $\ldots$ & 0 & 0 & $a$ \\
\hline
\end{tabular}

and the second, $\mathbf{A}_{\mathbf{n}+\mathbf{1}, \mathbf{a}_{\mathbf{n}+\mathbf{1}}}$, in which $\neg a_{1} \rightarrow a_{n+1}=a_{n+1}$ : 


\begin{tabular}{|c||c|c|c|c|c|c|c|c|c|c|c|c|}
\hline$\rightarrow$ & $a$ & $\neg a_{1}$ & $\neg a_{2}$ & $\ldots$ & $\neg a_{n}$ & $\neg a_{n+1}$ & $a_{n+1}$ & $a_{n}$ & $\ldots$ & $a_{2}$ & $a_{1}$ & $\neg a$ \\
\hline \hline$a$ & $a$ & $a$ & $a$ & $\ldots$ & $a$ & $a$ & $a_{n+1}$ & $a_{n-1}$ & $\ldots$ & $a_{2}$ & $a_{1}$ & $a_{1}$ \\
\hline$\neg a_{1}$ & 0 & $a$ & $a$ & $\ldots$ & $a$ & $a$ & $a_{n+1}$ & $a_{n+1}$ & $\ldots$ & $a_{3}$ & $a_{2}$ & $a_{1}$ \\
\hline$\neg a_{2}$ & 0 & 0 & $a$ & $\ldots$ & $a$ & $a$ & $a$ & $a$ & $\ldots$ & $a$ & $a_{3}$ & $a_{2}$ \\
\hline$\vdots$ & $\vdots$ & $\vdots$ & $\vdots$ & $\ddots$ & $\vdots$ & $\vdots$ & $\vdots$ & $\vdots$ & $\ddots$ & $\vdots$ & $\vdots$ & $\vdots$ \\
\hline$\neg a_{n}$ & 0 & 0 & 0 & $\ldots$ & $a$ & $a$ & $a$ & $a$ & $\ldots$ & $a$ & $a_{n+1}$ & $a_{n-1}$ \\
\hline$\neg a_{n+1}$ & 0 & 0 & 0 & $\ldots$ & 0 & $a$ & $a$ & $a$ & $\ldots$ & $a$ & $a_{n+1}$ & $a_{n+1}$ \\
\hline$a_{n+1}$ & 0 & 0 & 0 & $\ldots$ & 0 & 0 & $a$ & $a$ & $\ldots$ & $a$ & $a$ & $a$ \\
\hline$a_{n}$ & 0 & 0 & 0 & $\ldots$ & 0 & 0 & 0 & $a$ & $\ldots$ & $a$ & $a$ & $a$ \\
\hline$\vdots$ & $\vdots$ & $\vdots$ & $\vdots$ & $\ddots$ & $\vdots$ & $\vdots$ & $\vdots$ & $\vdots$ & $\ddots$ & $\vdots$ & $\vdots$ & $\vdots$ \\
\hline$a_{2}$ & 0 & 0 & 0 & $\ldots$ & 0 & 0 & 0 & 0 & $\ldots$ & $a$ & $a$ & $a$ \\
\hline$a_{1}$ & 0 & 0 & 0 & $\ldots$ & 0 & 0 & 0 & 0 & $\ldots$ & 0 & $a$ & $a$ \\
\hline$\neg a$ & 0 & 0 & 0 & $\ldots$ & 0 & 0 & 0 & 0 & $\ldots$ & 0 & 0 & $a$ \\
\hline
\end{tabular}

\section{Fundamental theorem}

Proposition. Each $\mathbf{A}_{\mathbf{n}}$-algebra is an $\mathbf{E}$-algebra.

We point out that we have two infinite sequences of algebras, i.e. a sequence $\mathbf{A}_{\mathbf{n}, \mathbf{a}}$ and the sequence $\mathbf{A}_{\mathbf{n}, \mathbf{a}_{\mathbf{n}}}$. In addition, none of these algebras have a proper subalgebra with the exception of the two-element subalgebra.

Each of these algebras is generated by the element $a$. Moreover, none of $\mathbf{A}_{\mathbf{n}}$-algebras have a non-trivial homomorphic image.

It entails the following theorem:

THEOREM 20. There exists two infinite sequences of finite simple $\boldsymbol{E}$-algebras such that the only proper subalgebra is $\mathbf{2}$.

Corollary 21. The interval $[\mathbf{E}, \mathbf{2}]$ has infinitely many coatoms.

REMARK. Note that for the logic RM there exist one pre-maximal extension and for the logic $\mathbf{R}$ there exist three pre-maximal extensions. 


\section{Another example of infinite sequences of E-algebras}

Let us consider the following lattice

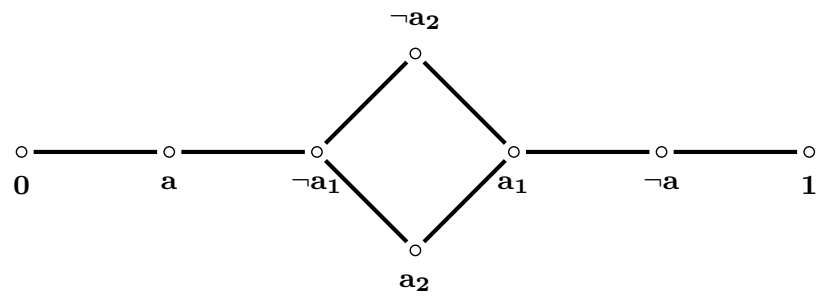

and an algebra based on this lattice. Of course, in this algebra the elements $a_{2}$ and $\neg a_{2}$ are not comparable. If we define the operation $\rightarrow$ as in $\mathbf{A}_{\mathbf{n}^{-}}$ algebras, then we get an $\mathbf{E}$-algebra.

In general, for the following lattice

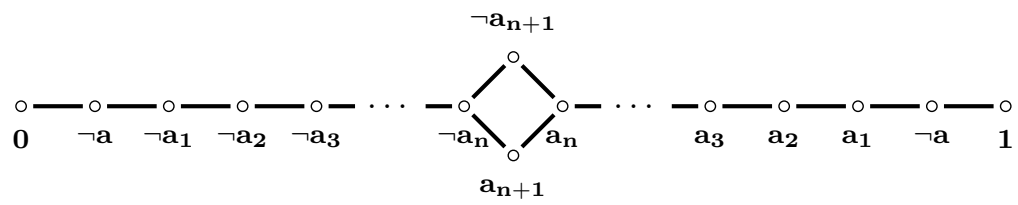

if we define the operation $\rightarrow$ as for the $\mathbf{A}_{\mathbf{n}}$-algebras, then we obtain an E-algebra.

\section{References}

[1] W. Ackermann, Begründung Einer Strengen Implikation, The Journal of Symbolic Logic, Vol. 21, 2 (1956), pp. 113-128.

[2] A. R. Anderson, N. D. Belnap, Jr., Entailment. The Logic of relevance and necessity, Princeton University Press, Vol. I (1975).

[3] N. D. Belnap, Jr., Intesional Models for First Degree Formula, The Journal of Symbolic Logic, Vol. 32, 1 (1967), pp. 1-22.

[4] W. J. Blok, D. Pigozzi, Algbebraizable logics, Memoirs of the American Mathematical Society, 1989.

[5] J. M. Font, G. B. Rodriguez, Note on algebraic models for relevance logic, Zeitschrift für Matematische Logik und Grundlagen der Mathematic, Vol. 36, 6 (1990), pp. 535-540. 
[6] W. Dziobiak, There are $2^{\aleph_{0}}$ Logics with the Relevance Principle Between $\mathbf{R}$ and RM, Studia Logica, Vol. XLII (1983), pp. 49-61.

[7] L. Maksimowa, Struktury s implikacjiej, Algebra and Logic, Vol. 12, 4 (1973), pp. 445-467.

[8] L. Maksimowa, O Modeljach iscislenija E, Algebra and Logic, Vol. 6, 6 (1967), pp. 5-20.

[9] R. M. Martin, Twenty-Third Annual Meeting of the Association for Symbolic Logic, The Journal of Symbolic Logic, Vol. 23, 4 (1958), pp. 456-461.

[10] R. K. Meyer, E and S4, Noter Dame Journal of Formal Logic, Vol. XI, 2 (1970), pp. 181-199.

[11] K. Świrydowicz, There exists exactly two maximal strictly relevant extensions of the relevant logic R, The Journal of Symbolic Logic, Vol. 64, 3 (1999), pp. 1125-1154.

[12] K. Świrydowicz, A Remark on the Maximal Extensions of the Relevant Logic $\boldsymbol{R}$, Reports on Mathematical Logic, 29 (1995), pp. 19-33.

[13] M. Tokarz, Essays in matrix semantics of relevant logics, The Institute of Philosophy and Sociology of the Polish Academy of Sciences, Warsaw 1980.

Acknowledgments. We would like to thank the anonymous referees for their comments.

Collegium Da Vinci

Kutrzeby 10

61-719 Poznań, Poland

e-mail: lidia.typanska@cdv.pl 